Review

\title{
Systems Molecular Imaging: Right Around the Corner
}

\author{
Baozhong Shen ${ }^{1,2,}$ \\ ${ }^{1}$ Department of Radiology, the Fourth Hospital of Harbin Medical University, Harbin, Heilongjiang, China \\ ${ }^{2}$ Molecular Imaging Center of Harbin Medical University, Harbin, Heilongjiang, China \\ Corresponding author: Baozhong Shen, Department of Radiology, the Fourth Hospital of Harbin Medical University, 31 Yinhang \\ Street, Nangang District, Harbin 150001, Heilongjiang, P. R. China. \\ Tel/Fax: +86-451-82576508; 86-451-82576509Ｅ-mail: shenbzh@vip.sina.com
}

Received: Dec. 20, 2013; Accepted: Jan. 17, 2014; Published: Jan. 20, 2014.

Citation: B. Z. Shen. Systems Molecular Imaging: Right Around the Corner. Nano Biomed. Eng. 20I4, 6(I), I-6.

DOI: $10.5101 /$ nbe.v6il.pI-6.

\begin{abstract}
With the development of 'omics', systems biology and molecular imaging technology, a common tendency of integration of a variety of multi-modality, multi-target imaging and theranostics technology has led to the establishment of a concept that could be called "systems molecular imaging". It can be used to show the complexity, diversity and in vivo biological behavior and the development and progress of disease in an organism qualitatively and quantitatively at a systems level. Ultimately, systems molecular imaging should enable the physicians not only to diagnose tumors accurately but also to provide 'on the spot' treatment efficiently. It will become comprehensive research tools and technical means for life science and medical sciences.
\end{abstract}

Keywords: System molecular imaging; Multifunctional nanoprobes; Theranostics; Multimodality imaging; Targeted imaging

\section{Introduction}

Due to the emergence of "omics" (e.g. function genomics, proteomics, pharmacogenomics) and the application of systems biology, as well as the advance in medical imaging technology, molecular imaging is now moving from speculation to clinical application. In 1999, Professor Ralph Weissleder from Harvard University first introduced the concept of "Molecular Imaging", a discipline that studies the biological process both qualitatively and quantitatively at cellular and molecular level using in vivo imaging technologies [1]. Molecular imaging is a result of the merging of molecular biology and modern radiology. It is of great importantance to the exploration of mechanism and development of diseases, to the evaluation of medication efficacy. It bridges basic life sciences and clinical medicine [2].

However, with the development of systems

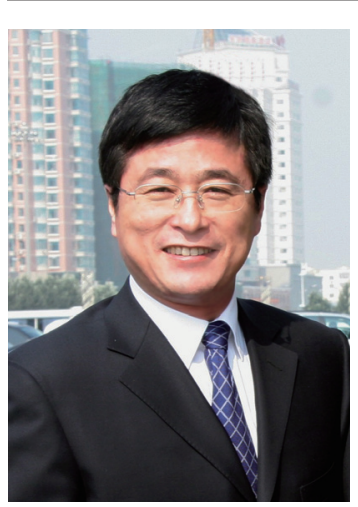

Baozhong Shen, M.D., Ph. D., the Professor of Medical Imaging and Nuclear Medicine in Harbin Medical University. His research team is developing imaging assays to monitor fundamental cellular/ molecular events in living subjects. His research interests include the biology and gene therapy of cancer as well as cardiovascular disease, early cancer detection, and pharmacological therapy assessment. He is also extending many of these approaches for human clinical applications using MR and PET-CT technologies. 
biology theory and technology, and further in depth understanding of human disease, the molecular imaging techniques targeting single biomarker, molecule, gene or protein are unable to meet the current needs of life science and medical science research. For example, single-modality molecular imaging is insufficient to acquire multiple information recognition of certain biomarker with the high sensitivity, because of the complexity of the pathophysiological processes, it is not possible to precisely characterize an intricate and dynamically changing molecular target of diseases by single-target molecular imaging (such as disease molecular classification); the specific change of an early stage disease can only be revealed by intelligent imaging technique. So to disclose and diagnose diseases; single molecular imaging method cannot be used to synchronously judge the effect of therapeutic interventions and determine treatment outcomes.

Based on original molecular imaging technology, we put forward the new concept, systems molecular imaging. The molecular imaging technique system can be applied to intuitively and systemically investigate the complicated biological network of a body and its functional process, by dynastically visualization of multiple key biomarkers in vivo molecular events of different level, and sometime execute interventions. Different from molecular imaging of single target, single mode and single imaging function, systems molecular imaging targets multiple biomarkers of different systems and series molecular events of biological processes. It combines a variety of imaging methods to achieve qualitative and quantitative identification, intervention and monitoring function and so on. It is necessary to explain that the "system" mentioned here is not the traditional histological anatomy term, but in accordance with the "system" concept defined in systems theory, namely an organic whole with certain functions consists of the relevant elements interconnected in a certain way. For example, in a tumor, the tumor cells, intra and inter-cell signaling pathways, stromal cells, and the microenvironment make up the tumor system.

The idea of system molecular imaging originated from systems biology [3] - in the hope of intuitively and comprehensively learning and understanding the normal interrelations among the different parties in the process of physiological events, their interactions (e.g., cell signal transduction pathways, cell organelles, cells, physiological systems and biological process related gene and protein network) and the related molecular events in the pathophysiological process of disease development and progression. Following the system theory and by means of molecular imaging probes synthesis and optimization, data mining and bioinformatics computing, it is an achievable goal to develop high sensitive multimodal molecular imaging system that can visualize multi-biomarker and multilevels molecular events in the biological process, to generate intellectualized imaging tools to sensitively monitor the biological responses in a certain unique microenvironment and to set up multi-function molecular imaging method to carry out targeted intervention and treatment effect monitoring at the same time.

\section{The advantages of system molecular imaging}

It is indicated in systems biology that biological behavior and the development and progress of disease in an organism is a very complex network process. Systems molecular imaging can provide comprehensive research tools and technical means for the life science and medical sciences. In the field of life science, systems molecular imaging can intuitively visualize the complicated biological network and physiological change, and significantly promote the development of systems biology. As for diseases, for example, the development, progression and metastasis of tumor is a very complicated dynamic process of molecular events that involves multi-steps, multi-stages and multi-targets [4]. It is associated with signal transduction from the surface of tumor cells, the interactions between tumor cells, and between tumor cells and tumor stroma, as well as the surrounding microenvironment, the interplay of tumor cells and immune cells and so on [5]. Although previous molecular imaging technology can visualize the critical target that plays an important role in progress of disease, it is impossible to exactly reflect the complicated biological behavior and characters of tumor merely by the visualization of a single molecular biomarker [6].

System molecular imaging technology can target various biological molecules and signal pathways that are important to development, progression, and change of tumor, and comprehensively reveal the interplay between different targets and signal transduction pathways and their dynamic changes, and so to accurately diagnose disease and evaluate therapeutic effect. 


\section{Systems molecular imaging and multi-pathways and multi-targets imaging}

The heterogeneity of tumor is a critical problem in terms of molecular classification and molecular targeted therapy [7-10]. Tumor heterogeneity shows in three aspects, individual heterogeneity, temporal heterogeneity and spatial heterogeneity. The molecular pathology examination is relatively unilateral, and it is difficult to deliver the accurate in vivo information of molecular target of in situ tumor. In vivo multitarget detection technology is needed to be carried out imperatively [11]. Gerlinger $\mathrm{M}$ et al. did molecular pathology and molecular typing on 101 tissue samples randomly from different parts of the tumors, differences in molecular typing was found in $63 \%$ to $69 \%$ region, which indicated that molecular pathology is not able to comprehensively show the molecular characteristics of tumor because of limitation of sampling [4]. In addition, molecular pathology can only reflect the status of a molecular target of a certain time point due to difficulty of repeated biopsy. But the critical tumor biomarkers and molecular types are always changing dynamically during tumorigenesis, development and therapy. Rosell R et al. treated 174 lung cancer patients with tyrosine kinase inhibitor erlotinib and found that the molecular type of tumors changed from molecular targeted drug sensitive to drug-resistance due to a secondary mutation (deletion mutation of EGFR exon 19 to T790M mutation), which directly results in the failure of molecular targeted drug therapy [12]. Thus, it's critically important to dynamically monitor the molecular biomarkers and tumor molecular type. Researchers had been wondering how to develop a personalized treatment for heterogeneous and continuously evolving active tumor. Only when we understand and quantify these changes, can it be possible to know the prognosis of tumor much more comprehensively, and to develop new effective therapeutic strategies. Systems molecular imaging can solve this issue, as it integrates multi-modal, multitargeted molecular imaging and theranostics imaging techniques. It can not only provide anatomical, functional and molecular information, but also disclose the biological relevance of different signal transduction pathways and molecular events by in vivo imaging.

So the open question is how to image the multiple targets in multiple signaling pathways. Here, we take tumor signaling pathways as an example, it has been known that EGFR, HER2, VEGFR, Integrin, HIF-1 and other targets in tumor cells play important roles on tumordevelopment and metastasis [13-17], the probes targeting these molecular signaling pathways have been developed [2]. For the EGFR signaling pathway, researchers used Cy5.5 to label modified hEGF, a natural ligand for EGFR. The synthetic EGF-Cy5.5 probe can target and visualize EGFR receptor extracellular binding domains [18-20]. Other investigators used radionuclide fluorine-18 or I-125 to labeled EGFR receptor tyrosine kinase inhibitor to target and image the mutated EGFR intracellular tyrosine activation domain $[21,22]$. Some others targeted the EGFR downstream phosphorylation signaling pathways, upregulated MDM2 messenger RNA [23], microRNA (microRNA-21) [24] and HIF-1 after phosphorylation and studied the microenvironment hypoxic events [25]. For HER2 receptor, Gao J et al. developed a non-toxic quantum probe for in vivo HER2 fluorescence imaging [26], Capala J et al. reported the recent progress of HER2targeted probes using trastuzumab, pertuzumab or a small molecule scaffold protein (e.g., affibody molecules) [27]. And for angiogenesis signaling pathways, Sipkins DA et al. utilized paramagnetic coupled contrast agents LM609 to target $\alpha_{v} \beta_{3}$ on endothelial cells to realized MR targeted molecular imaging [28]. Schmieder AH et al. used paramagnetic nanoparticles to target $\alpha_{v} \beta_{3}$ and achieve the real-time imaging of integrin and monitor its dynamical change in process of tumorigenesis and pulmonary embolism $[29,30]$. Sun $X$ et al. visualized $\alpha_{\mathrm{v}} \beta_{3}$ expression with constructing $18 \mathrm{~F}-\mathrm{FPPRGD} 2$ specific vascular molecular probes [31].

\section{Systems molecular imaging and multi-modalities imaging}

Clinicians have always been puzzled by choosing of the best clinical imaging modality for cancer patients. Normally, the sensitivity of the equipment with the highest resolution is relatively low and vice versa. How to "have it both ways" is a problem to be solved in the development of systems molecular imaging equipment field. Each imaging modality has its own advantages and disadvantages. Due to limitations of sensitivity, specificity and resolution, it is difficult to get systematic and comprehensive picture of the disease characteristics with a single imaging modality. For 
example, MRI based on magnetic nanoparticles can be used as an imaging tool to get oncology anatomical information (such as tumor volume and location), but cannot get the in vivo microscopic information, such as the internalization of magnetic nanoparticles, specific tumor molecular types and therapeutic efficacy at the cellular level, or the exact boundaries of residual tumor after surgical resection. However, optical imaging (e.g. fluorescence imaging) may satisfy the above goals [32]. With the integration of the anatomical and functional imaging modalities (such as CT, MRI, PET and optical) appears [33], multi-modality imaging combines the advantages of various imaging modalities (e.g., nuclear medicine with labeling various radioactive nuclides, positron tracers, SPECT tracers, imaging sequences of MR, optical, photoacoustic and ultrasound) to provide the anatomical, functional, molecular information, thus make imaging on the same object in different modality equipments possible [32]. Recently, researchers have developed a number of magnetic nanoparticles that can also be used for optical imaging [34-36]. These optical active magnetic nanoparticles can be synthesized by coupling fluorescent dye to the nanoparticles, or by using inorganic material-wrapped (e.g. gold, quantum dots, etc.) magnetic nanoparticles to synthesize the nanoparticles with surface plasmon resonance characteristics. Moreover, triple function reagents have been developed that can be used for PET, MRI and fluorescence imaging [37, 38]. Multi-modality magnetic nanoparticles can provide clinicians with powerful probes for tumor diagnosis and monitoring treatment effect.

\section{Systems molecular imaging in theranostics and drug development}

With the development of nanoscience and nanotechnology, the "image-guided drug delivery" (IGDD) and "Theranostics" technology of systems molecular imaging are promising to provide a novel and more effective tool for early diagnosis, imagining and treatment. IGDD is one of the advantages of the systems molecular imaging techniques in drug research and development,it maximize the therapeutic effect of agents on lesions and to minimize the toxic side effects by reducing systemic drug exposure [39, 40]. For example, multi-target combined therapy is one of the focuses of anti-cancer research, because patients with the same type tumor may require different targeting inhibitors. How can we know which molecular targeted inhibitor is the best for each patient? The systems molecular imaging techniques can display not only whether the targeted drugs are effectively targeting or delivered to the expected targets and released in local tumor or lesion, but also demonstrate the effect of the drugs on other related targets and signaling pathways disclosed by multi-targets imaging. Thus it can help to understand the pharmaceutical mechanism of specific biomarker targeting drug, and its the effects on biological systems, to select the appropriate population to the drug, and to analyze whether the patient is suitable for a specific treatment more comprehensively [41].

Theranostics is an integration of diagnosis and therapy techniques, which is another new trend of molecular imaging. This technology brings the imaging capabilities into the design of nanoparticles, to achieve real-time monitoring biodistribution of nanoparticles in individual patients and to develop personalized treatment protocol by adjusting the particle pharmacokinetics and uptake $[42,43]$. Theranostics can improve diagnosis, deliver drug specifically to the tumor, and reduce injury to normal tissue [44]. It can also provide information on the mechanism of the effect of nanoparticles, for example, the distribution, clearance mechanism of nanoparticles in the tumor can be monitored by a longitudinal dynamic imaging. It help us to understand the specific uptake, the rate of uptake, the distribution of these materials in the heterogeneous tumor microenvironment, the clearance rate and pathway, as well as the mechanisms of nano anticancer drug resistance or sensitivity. The information is critically important for the patient and for nanoparticles anticancer drug therapy selection [45]. Theranostics technology shortens the treatment and improves treatment by saving more tedious steps. Theranostics nanoprobes, including various types of nanoshells, plasmonic nanobubbles and quantum dots, have unique physical and chemical properties which some small molecules normally do not have. These characteristics can be used for designing new cancer treatment. The emergence of new integrated nanoprobes brings hope for cancer treatment, however, there are some huge obstacles and challenges for the clinical application of these agents: 1) Effective delivery. Increase the uptake of nano-probes in tumor will enhance the killing of tumor cells, reduce the damage to normal tissue, and increase the therapeutic window. 2) Image guidance. Real-time imaging is crucial for the design of cancer treatment and monitoring the 
efficacy. 3) Multi-mode treatment. Mono-therapy mode (including the application of nano-treatment probe) can not completely remove the residual tumor cells. Therefore, integrated a variety of treatment modes into a nano-particle system will be very promising [43].

To integrate multi-modality, multi-targets imaging and theranostics technology in the drug development process, scientists can quantitatively evaluate the function of small molecules, biological products, antibodies, proteins, ligands, gene therapy and vaccines at anatomical, functional and molecular level, which can screen potential candidate drugs, test new biomarkers, determine and optimize the therapeutic regimen and evaluate medical imaging equipment, new imaging probes and the imaging strategies in clinical trials. Researchers can also use this new technology: 1. To evaluate candidate drugs on living disease related and disease prevention animal model; 2. To comprehensively monitor the process of disease progression from the multiple related systems; 3 . Besides observation of the dynamic changes of related target, to assess the impact of drug side effect on the whole system, provide detailed data to enhance the decision-making confidence; 4 . To quantify experiment endpoint for clinical translation and beyond.

It is true there is foregoing "could," "would," and "potential" when describing systems molecular imaging, same as a decade ago when the emergence of molecular imaging. However, the potential successful integration of a variety of multi-modality, multi-targets imaging and theranostics technology is definitely encouraging and give credibility to expectations and theories. It's far beyond imagination and will certainly expand with the advancing of molecular imaging technology.

\section{Acknowledgement}

This paper is supported by the National Natural Science Foundation of China (81130028, 31210103913, 81225010), the Key Grant Project of Heilongjiang Province (GA12C302) and the Ph.D. Programs Foundation of Ministry of Education of China (201123071100203), and Chinese 973 Project (Grant No. 2010CB933901) and 863 Key Project (Grant No. 2007AA022004).

\section{References}

[1] Weissleder R: Molecular imaging: exploring the next frontier. Radiology 1999, 212(3): 609-614.
[2] Shen B: Molecular Imaging. Beijing: People's Health Publishing House (First Edition) 2007.

[3] Brazma A, Krestyaninova M, Sarkans U: Standards for systems biology. Nature reviews Genetics 2006, 7(8): 593-605.

[4] Gerlinger M, Rowan AJ, Horswell S, Larkin J, Endesfelder D, Gronroos E, Martinez P, Matthews N, Stewart A, Tarpey P et al.: Intratumor heterogeneity and branched evolution revealed by multiregion sequencing. The New England journal of medicine 2012, 366(10): 883-892.

[5] Quail DF, Joyce JA: Microenvironmental regulation of tumor progression and metastasis. Nature medicine 2013, 19(11): 1423-1437.

[6] Hanahan D, Weinberg RA: Hallmarks of cancer: the next generation. Cell 2011, 144(5): 646-674.

[7] Bedard PL, Hansen AR, Ratain MJ, Siu LL: Tumour heterogeneity in the clinic. Nature 2013, 501(7467): 355364.

[8] Marte B: Tumour heterogeneity. Nature 2013, 501(7467): 327.

[9] Meacham CE, Morrison SJ: Tumour heterogeneity and cancer cell plasticity. Nature 2013, 501(7467): 328-337.

[10] Murtaza M, Dawson SJ, Tsui DW, Gale D, Forshew T, Piskorz AM, Parkinson C, Chin SF, Kingsbury Z, Wong AS et al: : Non-invasive analysis of acquired resistance to cancer therapy by sequencing of plasma DNA. Nature 2013, 497(7447): 108-112.

[11] Sawyers CL: The cancer biomarker problem. Nature 2008, 452(7187): 548-552.

[12] Rosell R, Carcereny E, Gervais R, Vergnenegre A, Massuti B, Felip E, Palmero R, Garcia-Gomez R, Pallares C, Sanchez JM et al.: Erlotinib versus standard chemotherapy as first-line treatment for European patients with advanced EGFR mutation-positive non-smallcell lung cancer (EURTAC): a multicentre, open-label, randomised phase 3 trial. The lancet oncology 2012, 13(3): 239-246.

[13] Hynes NE, Lane HA: ERBB receptors and cancer: the complexity of targeted inhibitors. Nature reviews Cancer 2005, 5(5): 341-354.

[14] Roda JM, Sumner LA, Evans R, Phillips GS, Marsh CB, Eubank TD: Hypoxia-inducible factor-2alpha regulates GM-CSF-derived soluble vascular endothelial growth factor receptor 1 production from macrophages and inhibits tumor growth and angiogenesis. J Immunol 2011, 187(4): 1970-1976.

[15] Ho QT, Kuo CJ: Vascular endothelial growth factor: biology and therapeutic applications. The international journal of biochemistry \& cell biology 2007, 39(7-8): 1349-1357.

[16] Ferrara N: Vascular endothelial growth factor: basic science and clinical progress. Endocrine reviews 2004, 25(4): 581-611.

[17] Semenza GL: Targeting HIF-1 for cancer therapy. Nature reviews Cancer 2003, 3(10): 721-732.

[18] Zhang L, Wang K, Zhao F, Hu W, Chen J, Lanza GM, Shen B, Zhang B: Near Infrared Imaging of EGFR of Oral Squamous Cell Carcinoma in Mice Administered Arsenic Trioxide. PloS one 2012, 7(9): e46255.

[19] Wang K, Wang K, Li W, Huang T, Li R, Wang D, Shen B, Chen X: Characterizing breast cancer xenograft epidermal growth factor receptor expression by using near-infrared optical imaging. Acta Radiol 2009, 50(10): 1095-1103.

[20] Ke S, Wen X, Gurfinkel M, Charnsangavej C, Wallace S, Sevick-Muraca EM, Li C: Near-infrared optical imaging of epidermal growth factor receptor in breast cancer xenografts. Cancer Res 2003, 63: 7870-7875.

[21] Hirata M, Kanai Y, Naka S, Yoshimoto M, Kagawa S, Matsumuro K, Katsuma H, Yamaguchi H, Magata 
Y, Ohmomo Y: A useful EGFR-TK ligand for tumor diagnosis with SPECT: development of radioiodinated 6-(3-morpholinopropoxy)-7-ethoxy-4-(3'-iodophenoxy) quinazoline. Annals of nuclear medicine 2013, 27(5): 431-443.

[22] Yeh HH, Ogawa K, Balatoni J, Mukhapadhyay U, Pal A, Gonzalez-Lepera C, Shavrin A, Soghomonyan S, Flores L, 2nd, Young D et al.: Molecular imaging of active mutant L858R EGF receptor (EGFR) kinase-expressing nonsmall cell lung carcinomas using PET/CT. Proceedings of the National Academy of Sciences of the United States of America 2011, 108(4): 1603-1608.

[23] Fu P, Shen B, Zhao C, Tian G: Molecular imaging of MDM2 messenger RNA with $99 \mathrm{mTc}$-labeled antisense oligonucleotides in experimental human breast cancer xenografts. J Nucl Med 2010, 51(11): 1805-1812.

[24] Zhao D, Tu Y, Wan L, Bu L, Huang T, Sun X, Wang K, Shen B: In Vivo Monitoring of Angiogenesis Inhibition via Down-Regulation of Mir-21 in a VEGFR2-Luc Murine Breast Cancer Model Using Bioluminescent Imaging. PLoS One 2013, 8(8): e71472.

[25] Huang T, Civelek AC, Li J, Jiang H, Ng CK, Postel GC, Shen B, Li XF: Tumor microenvironment-dependent 18F-FDG, 18F-fluorothymidine, and 18F-misonidazole uptake: a pilot study in mouse models of human nonsmall cell lung cancer. $J$ Nucl Med 2012, 53(8): 12621268.

[26] Gao J, Chen K, Miao Z, Ren G, Chen X, Gambhir SS, Cheng Z: Affibody-based nanoprobes for HER2expressing cell and tumor imaging. Biomaterials 2011, 32(8): 2141-2148.

[27] Capala J, Bouchelouche K: Molecular imaging of HER2positive breast cancer: a step toward an individualized 'image and treat' strategy. Current opinion in oncology 2010, 22(6): 559-566.

[28] Sipkins DA, Cheresh DA, Kazemi MR, Nevin LM, Bednarski MD, Li KC: Detection of tumor angiogenesis in vivo by alphaVbeta3-targeted magnetic resonance imaging. Nat Med 1998, 4(5): 623-626.

[29] Schmieder AH, Winter PM, Williams TA, Allen JS, Hu G, Zhang H, Caruthers SD, Wickline SA, Lanza GM: Molecular MR Imaging of Neovascular Progression in the Vx2 Tumor with alphavbeta3-Targeted Paramagnetic Nanoparticles. Radiology 2013, 268(2): 470-480.

[30] Schmieder AH, Wang K, Zhang H, Senpan A, Pan D, Keupp J, Caruthers SD, Wickline SA, Shen B, Wagner $\mathrm{EM}$ et al.: Characterization of early neovascular response to acute lung ischemia using simultaneous F/ H MR molecular imaging. Angiogenesis 2013.

[31] Sun X, Yan Y, Liu S, Cao Q, Yang M, Neamati N, Shen B, Niu G, Chen X: 18F-FPPRGD2 and 18F-FDG PET of Response to Abraxane Therapy. J Nucl Med 2011, 52(1): 140-146.

[32] Weissleder R, Pittet MJ: Imaging in the era of molecular oncology. Nature 2008, 452(7187): 580-589.

[33] Kinahan PE, Townsend DW, Beyer T, Sashin D: Attenuation correction for a combined 3D PET/CT scanner. Medical physics 1998, 25(10): 2046-2053.

[34] Medarova Z, Pham W, Farrar C, Petkova V, Moore A: In vivo imaging of siRNA delivery and silencing in tumors. Nature medicine 2007, 13(3): 372-377.

[35] Lee H, Yu MK, Park S, Moon S, Min JJ, Jeong YY, Kang HW, Jon S: Thermally cross-linked superparamagnetic iron oxide nanoparticles: synthesis and application as a dual imaging probe for cancer in vivo. Journal of the American Chemical Society 2007, 129(42): 12739-12745.

[36] Foy SP, Manthe RL, Foy ST, Dimitrijevic S, Krishnamurthy N, Labhasetwar V: Optical imaging and magnetic field targeting of magnetic nanoparticles in tumors. ACS nano 2010, 4(9): 5217-5224.

[37] Xie J, Chen K, Huang J, Lee S, Wang J, Gao J, Li X, Chen X: PET/NIRF/MRI triple functional iron oxide nanoparticles. Biomaterials 2010, 31(11): 3016-3022.

[38] Stelter L, Pinkernelle JG, Michel R, Schwartlander R, Raschzok N, Morgul MH, Koch M, Denecke T, Ruf $\mathrm{J}$, Baumler $\mathrm{H}$ et al:: Modification of aminosilanized superparamagnetic nanoparticles: feasibility of multimodal detection using 3T MRI, small animal PET, and fluorescence imaging. Molecular imaging and biology : MIB : the official publication of the Academy of Molecular Imaging 2010, 12(1): 25-34.

[39] Scheffler M, Kobe C, Zander T, Nogova L, Kahraman D, Thomas R, Neumaier B, Dietlein M, Wolf J: Monitoring reversible and irreversible EGFR inhibition with erlotinib and afatinib in a patient with EGFR-mutated nonsmall cell lung cancer (NSCLC) using sequential [18F] fluorothymidine (FLT-)PET. Lung Cancer 2012, 77(3): 617-620.

[40] Shen B: Molecular Imaging. Beijing: People's Health Publishing House (Second Edition) 2010.

[41] Lanza GM, Moonen C, Baker JR, Jr., Chang E, Cheng Z, Grodzinski P, Ferrara K, Hynynen K, Kelloff G, Lee YE et al.: Assessing the barriers to image-guided drug delivery. Wiley Interdiscip Rev Nanomed Nanobiotechnol 2014, 6(1): 1-14.

[42] Horcajada P, Chalati T, Serre C, Gillet B, Sebrie C, Baati T, Eubank JF, Heurtaux D, Clayette P, Kreuz C et al.: Porous metal-organic-framework nanoscale carriers as a potential platform for drug delivery and imaging. Nature materials 2010, 9(2): 172-178.

[43] Shen B: Molecular Imaging: Principle and Practice. Beijing: People's Health Publishing House 2013.

[44] Palekar-Shanbhag P, Jog SV, Chogale MM, Gaikwad SS: Theranostics for cancer therapy. Current drug delivery 2013, 10(3): 357-362.

[45] Pan D, Sanyal N, Schmieder AH, Senpan A, Kim B, Yang X, Hu G, Allen JS, Gross RW, Wickline SA et al.: Antiangiogenic nanotherapy with lipase-labile Sn-2 fumagillin prodrug. Nanomedicine 2012, 7(10): 15071519.

Copyright $\odot 2014$ B.Z. Shen. This is an open-access article distributed under the terms of the Creative Commons Attribution License, which permits unrestricted use, distribution, and reproduction in any medium, provided the original author and source are credited. 